\title{
Differentiation of Eight Commercial Mushrooms by Electronic Nose and Gas Chromatography-Mass Spectrometry
}

\author{
Jinjie Zhou, ${ }^{1}$ Tao Feng, ${ }^{1}$ and Ran $\mathrm{Ye}^{2}$ \\ ${ }^{1}$ School of Perfume and Aroma Technology, Shanghai Institute of Technology, No. 100 Hai Quan Road, Shanghai 201418, China \\ ${ }^{2}$ Department of Biosystems Engineering and Soil Science, University of Tennessee, 2506 E.J. Chapman Drive, Knoxville, \\ TN 37996-4531, USA
}

Correspondence should be addressed to Tao Feng; fengtao@sit.edu.cn and Ran Ye; rye@utk.edu

Received 14 October 2014; Revised 30 November 2014; Accepted 1 December 2014

Academic Editor: Luisa Torsi

Copyright (C) 2015 Jinjie Zhou et al. This is an open access article distributed under the Creative Commons Attribution License, which permits unrestricted use, distribution, and reproduction in any medium, provided the original work is properly cited.

\begin{abstract}
Volatile profiles of eight mushrooms were characterized by gas chromatography-mass spectrometry and electronic nose analysis. Volatile compounds including 11 alcohols, 11 ketones, 15 aldehydes, 3 sulfur compounds and alkenes, 8 terpenes, 7 acid and esters, 5 heterocyclic compounds, 20 aromatic compounds, and 4 other compounds were identified. The overall aroma properties of the mushrooms were analyzed by the electronic nose. Results indicated that the e-nose sensors have the ability to accurately respond to different mushrooms with similar fingerprint chromatograms. The relationship between the GC-MS data and e-nose responses of different mushrooms was modeled by principal component analysis and partial least squares regression. This combination for the volatile analysis with chemometric methods can be applied to distinguish different mushrooms successfully. Furthermore, it is concluded that the volatile composition of commercial mushrooms could benefit a finger spectrum by e-nose to identify the species of edible fungi.
\end{abstract}

\section{Introduction}

Mushrooms are fleshy and fruiting bodies containing a wide range of edible fungi, such as Lentinus edodes (shiitake), Pleurotus abalonus, Agrocybe aegirit, Hericium erinaceus, Pleurotus eryngii. Because of their attractive tastes, flavors, and nutritional characteristics, mushrooms are commonly used as food ingredients and also as one of the fundamental components in traditional Chinese medicines [1]. Generally, the specific pleasant odors of mushroom species and their products are described as almond-like or anise-like odors, floral or herb odors, or fruity odors [2]. For instance, the fruity flavor is typical of some species such as Armillaria mellea, Ceriporiopsis subvermispora, and Dichomitus squalens [3]. The fragrant flavor is achieved from Pleurotus sapidus and Stereum sanguinolentum, whereas the pleasant and anise are considered as characteristic flavors of Phaeolus schweinitzii and Gloeophyllum odoratum [2].

Although the quality of mushrooms is highly associated with numerous factors including aroma, taste, color, and texture, the aroma of the mushroom plays a major role in sensory attributes and consumer acceptance [1]. Because the unique mushroom flavors correspond to species, this could be employed to discriminate different mushroom species [4]. As one of the main compounds accounting for the unique mushroomy flavor, 1-octen-3-ol was first discovered in Tricholoma matsutake [5], and subsequently a series of C8 aliphatic components was reported to be responsible for the mushroom flavor, such as 3-octanol and 3-octanone [6]. Gas chromatography-mass spectrometry (GC-MS), gas chromatograph-flame ionization detector (GC-FID), and headspace-gas chromatograph (HS-GC) analysis have been widely applied in the analysis of mushroom volatile components [7]. Currently, approximately 150 different volatile compounds have been identified in mushrooms and classified into several categories such as alcohols, aldehydes, alkanes, aromatics, sulphur compounds, lower terpenes, and others [8]. Malheiro et al. [9] demonstrated the potential of using volatile components to discriminate six mushroom species, using GC-MS combined with e-nose. 
Sensory evaluation is a common method in the flavor analysis of foods. However, there are a number of disadvantages in sensory evaluation including high cost of training panelists, panelist subjectivity, incapacity of online monitoring, and time-consuming. As an alternative approach, electronic nose (e-nose) combining with GC-MS is an innovative and emerging technology for odor analysis with the powerful capability in qualitative and quantitative determination of trace volatile components in food samples [20]. This method exhibits great advantages such as rapid detection, high objectivity, high sensitivity (suitable for tiny amount of samples), long-term routine application, simplicity, and ease of use. Feng et al. [21] analyzed the volatile compounds of Mesona Blumes gum/rice extrudates using GC-MS and enose, and the results showed that this was able to effectively distinguish Mesona Blumes gum/rice (MBG) extrudates at different MBG content. Wang et al. [20] also demonstrated that the e-nose sensors combining with GC-MS were capable of clearly and rapidly distinguishing the flavor differences among synthetic milk, natural milk, and the enzyme-induced milk. Furthermore, this method has been also utilized in the identification of the geographical origin of propolis [22].

Traditionally, e-nose can be just used as a discrimination tool to differentiate various samples. However, it still suffers from detailed information regarding the difference between the discriminated samples. It is well known that sensors on e-nose could have different stimuli to different chemical compounds, which might be used as a typical approach to correlate the chemical compounds and sensors of e-nose. Therefore, it can be used as a finger spectrum to characterize the concrete chemical compound. However, little information has been reported in odor analysis of mushrooms by GC-MS and e-nose. The major objectives of this work were to (1) study the feasibility of electronic nose sensors for discriminating the different mushrooms; (2) investigate the volatile compositions of mushrooms using GC-MS analysis; (3) conduct the correlation analysis between aroma compounds and electronic nose responses for the interpretation of sensor properties using multivariate analysis of principal component analysis (PCA) and partial least squares regression (PLSR).

\section{Materials and Methods}

2.1. Materials. The eight dried commercial edible mushrooms of Pleurotus abalonus, Agrocybe aegirit, Hericium erinaceus, Grifola frondosa, Coprinus comatus, Boletus edulis, Lentinula edodes, Pleurotus eryngii were purchased from a supermarket of Tesco, Shanghai, China. The species of the mushrooms were identified by the manufacturers and labeled in the package bags. After arrival, the samples were redried at an $80^{\circ} \mathrm{C}$ oven for $4 \mathrm{~h}$ to achieve same moisture content. The dried mushrooms were crushed in a disintegrator (Dianjiu Traditional Medicine Machinery Manufacture Co. Ltd., Shanghai, China) and the powders were packaged in PVC bags and kept in a dry and dark place at $-18^{\circ} \mathrm{C}$ for further use.

Standard compounds of 1-octen-3-ol, nerolidol, spathulenol, cedrenol, 3-octanone, 2-octanone, isovaleraldehyde, hexanal, octanal, dimethyl trisulfide, furan, 2-pentyl-, 2ethyl-3,6-dimethylpyrazine, benzaldehyde, phenyl acetaldehyde, anethole, benzothiazole, ortho cresol, naphthalene, 2,6dimethyl-, 1,1'-biphenyl, 4-methyl-, $\alpha$-cubebene, cyperene, $\alpha$-copaene, methyl cinnamate, nonanoic acid, and $\beta$-ionone were purchased from Sigma-Aldrich (St. Louis, MO, USA). All other chemicals were of analytical reagent grade and were purchased from Sinopharm Chemical Reagent Co. Ltd., Shanghai, China. Ultrapure water was obtained from Watsons, Shanghai, China.

2.2. Preparation of Mushroom Extracts. The mushroom powders were sieved through 80 mesh griddles and about $25 \mathrm{~g}$ was transferred into a $2 \mathrm{~L}$ round bottom flask. Solvent of deionized water was added in the flask at a solid-liquid ratio of $1: 10$ and steam distilled for $2.5 \mathrm{~h}$. After cooling to ambient temperature, the distillation extract was collected and equal volume of anhydrous ethyl ether was added for extracting the flavor compounds. The extract was dried over anhydrous sodium sulphate, maintained at freeze temperature of $-18^{\circ} \mathrm{C}$ to remove water (as ice crystals), and then concentrated to $1 \mathrm{~mL}$ prior to further analysis.

\subsection{Volatile Compound Analysis}

2.3.1. GC-MS Analysis. GC-MS analysis was conducted using an Agilent $7890 \mathrm{~N}$ gas chromatography-5975 mass selective detector (GC-MS) (Agilent Technologies Inc., Palo Alto, CA), equipped with a HP-INNOWAX column $(60 \mathrm{~m} \times 0.25 \mathrm{~mm} \times$ $0.25 \mu \mathrm{m})$. The carrier gas was used as helium at a constant flow rate of $1.0 \mathrm{~mL} / \mathrm{min}$. The injector port was heated to $250^{\circ} \mathrm{C}$, using the splitless injection mode. The initial oven temperature was maintained at $40^{\circ} \mathrm{C}$ for $3 \mathrm{~min}$, then raised to $150^{\circ} \mathrm{C}$ at a rate of $5^{\circ} \mathrm{C} / \mathrm{min}$ and held for $1 \mathrm{~min}$, and finally raised to $220^{\circ} \mathrm{C}$ at a rate of $10^{\circ} \mathrm{C} / \mathrm{min}$ and maintained for $2 \mathrm{~min}$. The temperatures of injector and detector were $250^{\circ} \mathrm{C}$ and $220^{\circ} \mathrm{C}$, respectively. The mass spectra were captured in the electron impact (EI) ionization mode, with an ionization voltage of $70 \mathrm{eV}$ and a scanning range of $\mathrm{m} / z 40-400$. Other parameters included the ion source of $230^{\circ} \mathrm{C}$ and mass spectrometry interface of $280^{\circ} \mathrm{C}$. Each measurement was performed in triplicate and repeated three times.

\subsubsection{Identification and Quantification of Volatile Compounds.} The identification of volatile compounds was based on computer matching with the mass spectra in the NIST 05, WILEY and ADAMS libraries, as well as by comparison of the mass spectra and retention indices (RI) according to those reported in the literatures [1, 10-19, 23]. In addition, a home-made library in the Shanghai Institute of Technology, based on the analysis of reference oils and commercially available standards, was also used for the identification and quantification.

\subsection{Electronic Nose Analysis of Volatile Compounds}

2.4.1. The Preparation of the Sample for Electronic Nose. For e-nose analysis, the mushroom powders were sieved through 
40 mesh griddles. About $0.2 \mathrm{~g}$ powders was put into a $10 \mathrm{~mL}$ vial and kept in a chamber at controlled temperature $\left(37^{\circ} \mathrm{C}\right)$ and humidity (50\%) [24] for further use.

2.4.2. Electronic Nose Detection. An e-nose of AlphaMOS FOX 4000 (AlphaMOS, Toulouse, France) was applied to study the volatile compounds. The device was composed of eighteen metal-oxide sensors with a headspace autosampler HS100, e-nose unit, and e-nose software. 18 different metal oxide sensors could be divided into three chambers [25], which were three types of sensors, that is, six "LY" type sensors, five " $\mathrm{T}$ " type sensors, and seven " $\mathrm{P}$ " type sensors. The response characteristics of the gas sensors varied depending on their types [26]. Types $\mathrm{P}$ and $\mathrm{T}$ sensors are based on tin dioxide $\left(\mathrm{SnO}_{2}\right)$ but have different sensor geometries. LY sensors are chromium-titanium oxides $\left(\mathrm{Cr}_{2}-\mathrm{xTixO}_{3}-\mathrm{y}\right)$ and tungsten oxide $\left(\mathrm{WO}_{3}\right)$ sensors [25]. Various types of sensors were used in instruments to ensure sufficient sensitivity and selectivity.

Each sample vial was heated to $50^{\circ} \mathrm{C}$ and then agitated at $500 \mathrm{rpm}$ for $900 \mathrm{~s} \mathrm{immediately} \mathrm{prior} \mathrm{to} \mathrm{injection.} \mathrm{The} \mathrm{sample}$ headspace volume of $2.5 \mathrm{~mL}$ was drawn from the vial at $500 \mu \mathrm{L} / \mathrm{s}$, using a syringe maintained at $60^{\circ} \mathrm{C}$. The sample was injected into the e-nose at a speed of $500 \mu \mathrm{L} / \mathrm{s}$ and delivered to the sensors with a purified air carrier gas $\left(\mathrm{O}_{2}+\mathrm{N}_{2}>99.95 \%\right.$, $\left.\mathrm{O}_{2}=20 \pm 1 \%, \mathrm{H}_{2} \mathrm{O}<5 \mathrm{ppm}, \mathrm{CO}_{2}<5 \mathrm{ppm}, \mathrm{C}_{n} \mathrm{H}_{m}<5 \mathrm{ppm}\right)$ at a flow rate of $150 \mathrm{~mL} / \mathrm{min}$. Sensor resistances were recorded for $120 \mathrm{~s}$, and $600 \mathrm{~s}$ of delay was used to allow the sensor to return to baseline values before the next injection.

2.5. Statistical Analysis. The GC-MS profiles of mushroom were analyzed by PCA, and PLS2 was used to explain correlations among GC-MS and e-nose data sets. Partial least squares regression (PLSR) [25] were performed with GC-MS and e-nose data. For determining the predictability of e-nose sensors from GC-MS data, PLS1 regression was performed with GC-MS data as the $X$-variable and e-nose data as $Y$-variable. Regression coefficients were analyzed by jack-knifing.

All variables were centered and standardized to make each variable has a unit variance and zero mean before applying PLS analysis. All PLSR models were validated using full cross-validation. Statistical analysis was performed by using the Unscrambler v.9.7 (CAMO ASA, Oslo, Norway).

\section{Results and Discussion}

3.1. Volatile Compounds in Different Mushrooms. The volatile compounds in the mushrooms were extracted by steam distillation and then analyzed by GC-MS. Table 1 lists the tentatively identified 88 compounds in which 31 compounds were identified using the Wiley MS Library Database and 51 were identified by comparison of the retention time and the MS spectrum of the pure chemical standards. These include 11 alcohols, 11 ketones, 15 aldehydes, 3 sulfur compounds and alkenes, 8 terpenes, 8 acid and esters, 5 heterocyclic compounds, 20 aromatic compounds, and 4 other compounds.
Alcohols have been considered as the main odorants of the mushroomy aroma (Cho et al. $[1,8]$ ). In the present work, the alcohols with the high concentrations were detected in species of $P$. abalonus, L. edodes, and P. eryngii, followed by $H$. erinaceus (Figure 1). Among the alcoholic compounds, 1-octen-3-ol has the highest concentration in L. edodes (Table 1), whereas 3-octanol owns the highest concentration in $P$. abalonus $(\sim 11.6 \%)$. It has been reported that the C8 aliphatic compounds, including 1-octen-3-ol, 3-octanol, 3octen-2-one, and 2-octenal, 3-octanone, are the major contributors to the characteristic flavor of mushroom of Tricholoma matsutake [27]. These C8 compounds are mainly formed by the oxidation of linoleic or linolenic acids in the presence of enzymes of lipoxygenase and hydroperoxide lyase [28].

The results also indicated that $P$. abalonus, $A$. aegirit, and C. comatus contained the highest level of ketones (Figure 1), accounting for $\sim 26.0 \%, 19.2 \%$, and $18.1 \%$ of the total volatile compounds in these species, respectively. Ketones of 3octanone and 2-undecanone were identified in all of the tested species and P. abalonus consisted of the highest level compared to others (Table 1). The characteristic component of 3-octanone is a common herb aroma, and 2-undecanone is considered to be the main compound responsible for fruity flavor [29]. It is well-recognized that some odoractive ketone substances, such as $\beta$-ionone and trans-geranyl acetone, belong to oxidative by-products or degradation products derived from carotenoids (and therefore called norisoprenoids) and have been identified in mushrooms [30]. Ketones of $\beta$-ionone and $\beta$-dihydro-ionone are also important flavor compounds in some port wines [31]. In this work, geranyl acetone is the highest content in the mushrooms of $A$. aegirit and $C$. comatus, with the aroma description of green and magnolia, implying that this compound could be a flavor marker of these mushroom species.

Aldehydes were the third most representative chemicals in the tested mushrooms, with 15 compounds being identified. About $35.8 \%$ of the total flavor compounds in $A$. aegirit and about $48.2 \%$ in $H$. erinaceus were aldehydes (Figure 1). Among the identified aldehydes, hexanal (5.7\%), nonanal (7.2\%), 6-nonenal (4.2\%), and (2E, $4 \mathrm{E})-2$, 4-decadienal $(12.2 \%)$ had the highest concentration in the species of $P$. eryngii, C. comatus, $A$. aegirit, and $H$. erinaceus, respectively. In addition, octanal and (E)-2-octenal had the highest concentrations in $H$. erinaceus and P. eryngii (Table 1 ). Of interest, no aldehyde was detected in the mushroom of $L$. edodes. A homologous series of n-aldehydes from C-5 to C10 and simple unsaturated aldehydes from C-7 to C-10 were observed in the samples (Table 1). These compounds could be derived from the products of degradation or oxidation of the lipid in mushrooms [32]. (E)-2-Heptenal, 2-octenal, and (2E, 4E)-2, 4-decadienal was observed in all species except in L. edodes. It was suggested that the aldehydes of 5-methyl2-phenyl-2-hexenal, benzaldehyde, and phenyl acetaldehyde were generated from the Maillard reaction pathway [33]. Chen and $\mathrm{Wu}[16]$ also demonstrated the presence of 5methyl-2-phenyl-2-hexenal in mushroom of Agaricus subrufescens. Volatile compounds of aldehydes generally displayed coarse and heavy aromas of raw fish [34]. Different 


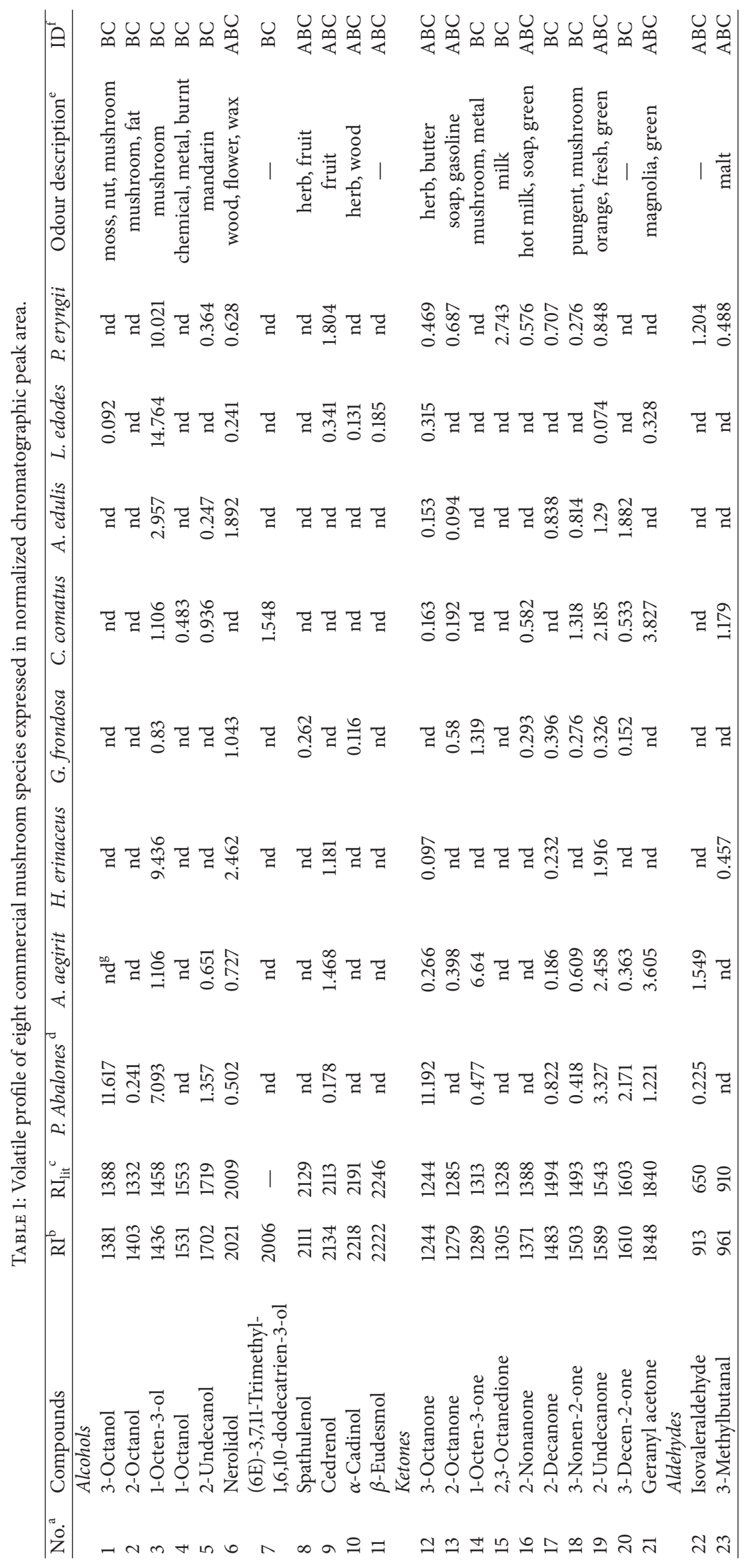




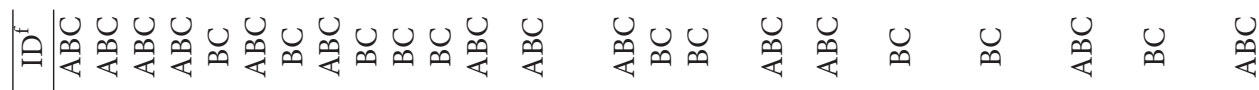

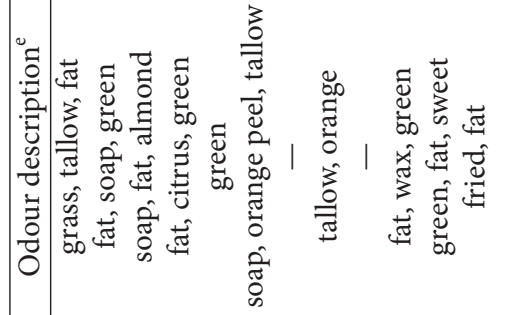

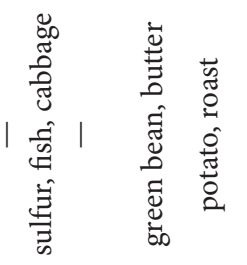

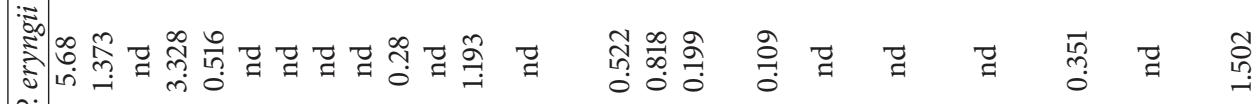
si

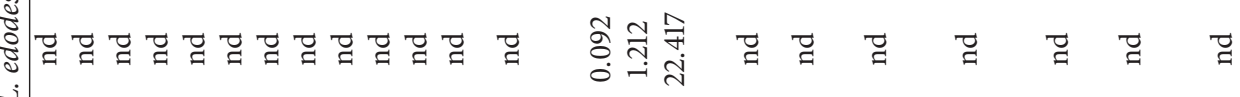

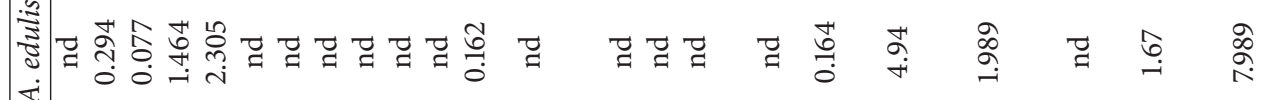

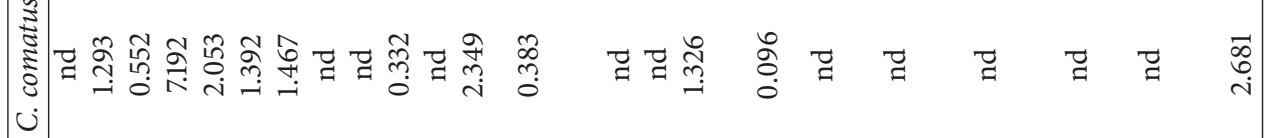

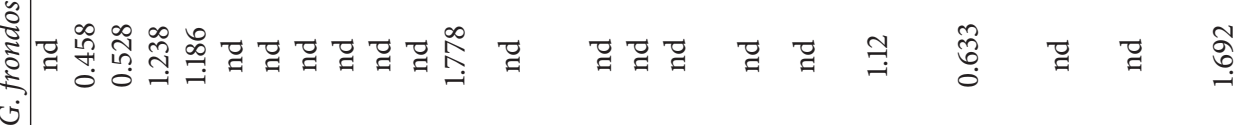
(3)

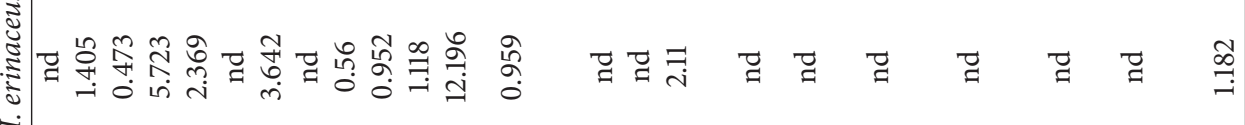
ذં

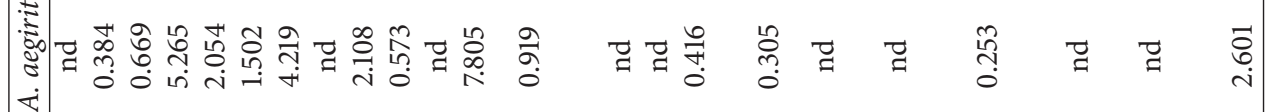

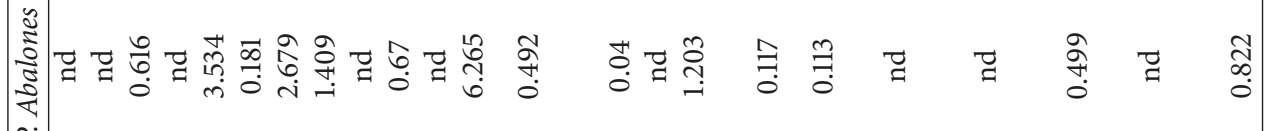
尺i

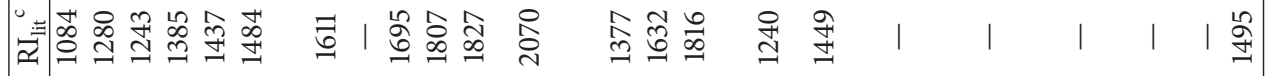

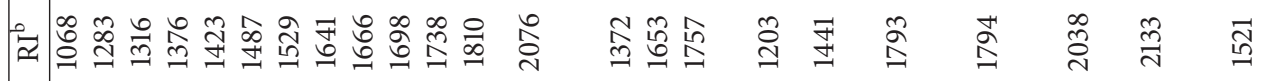

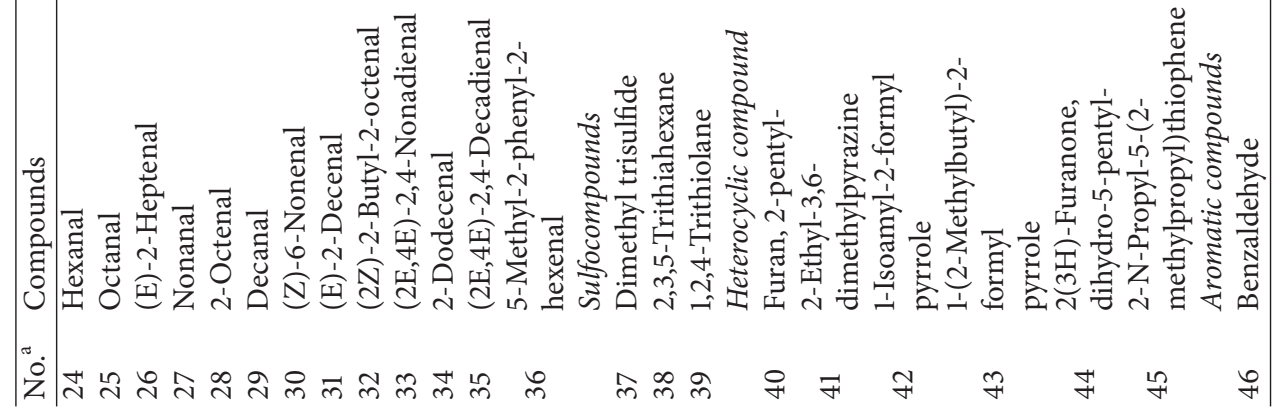




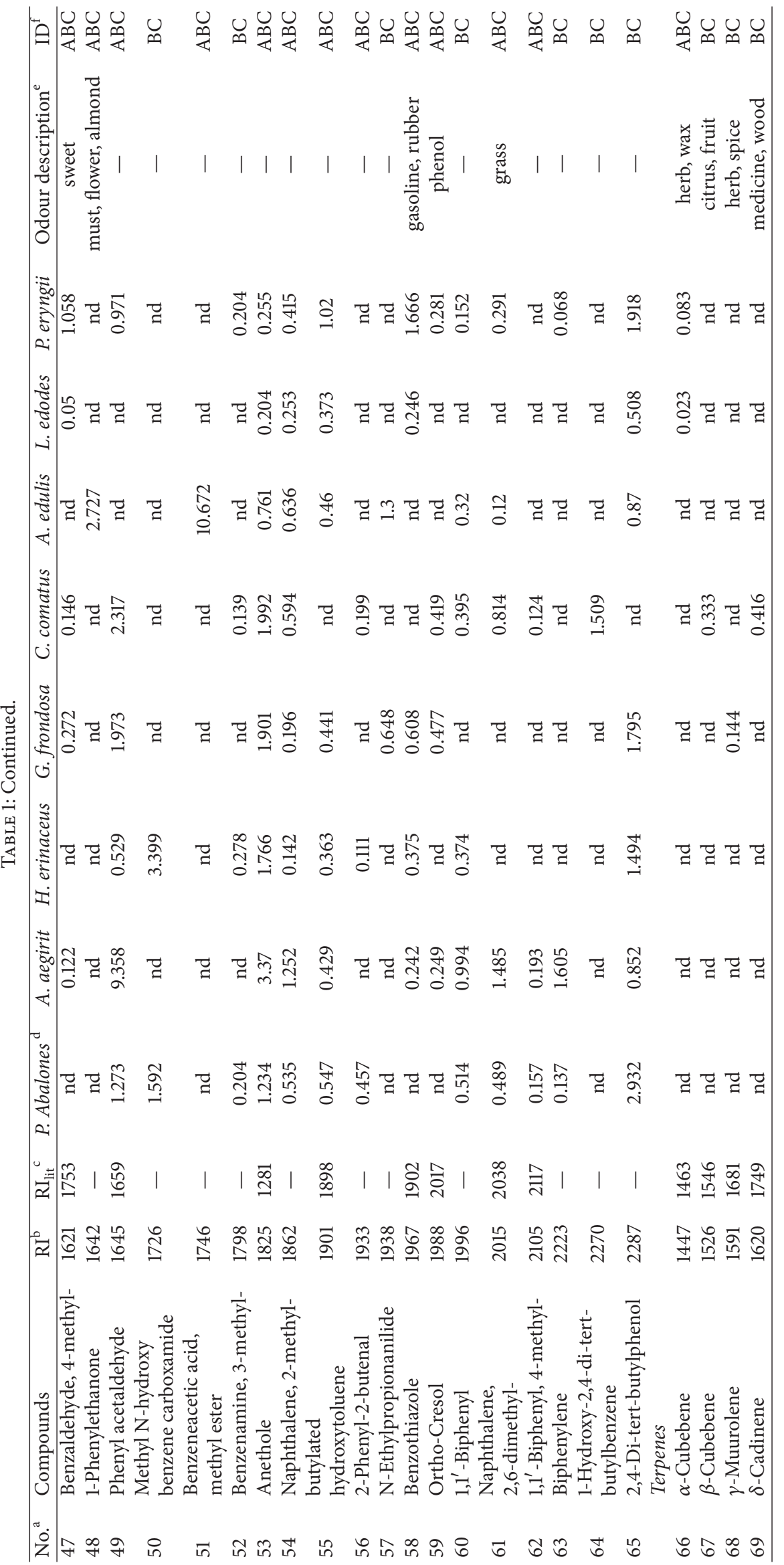




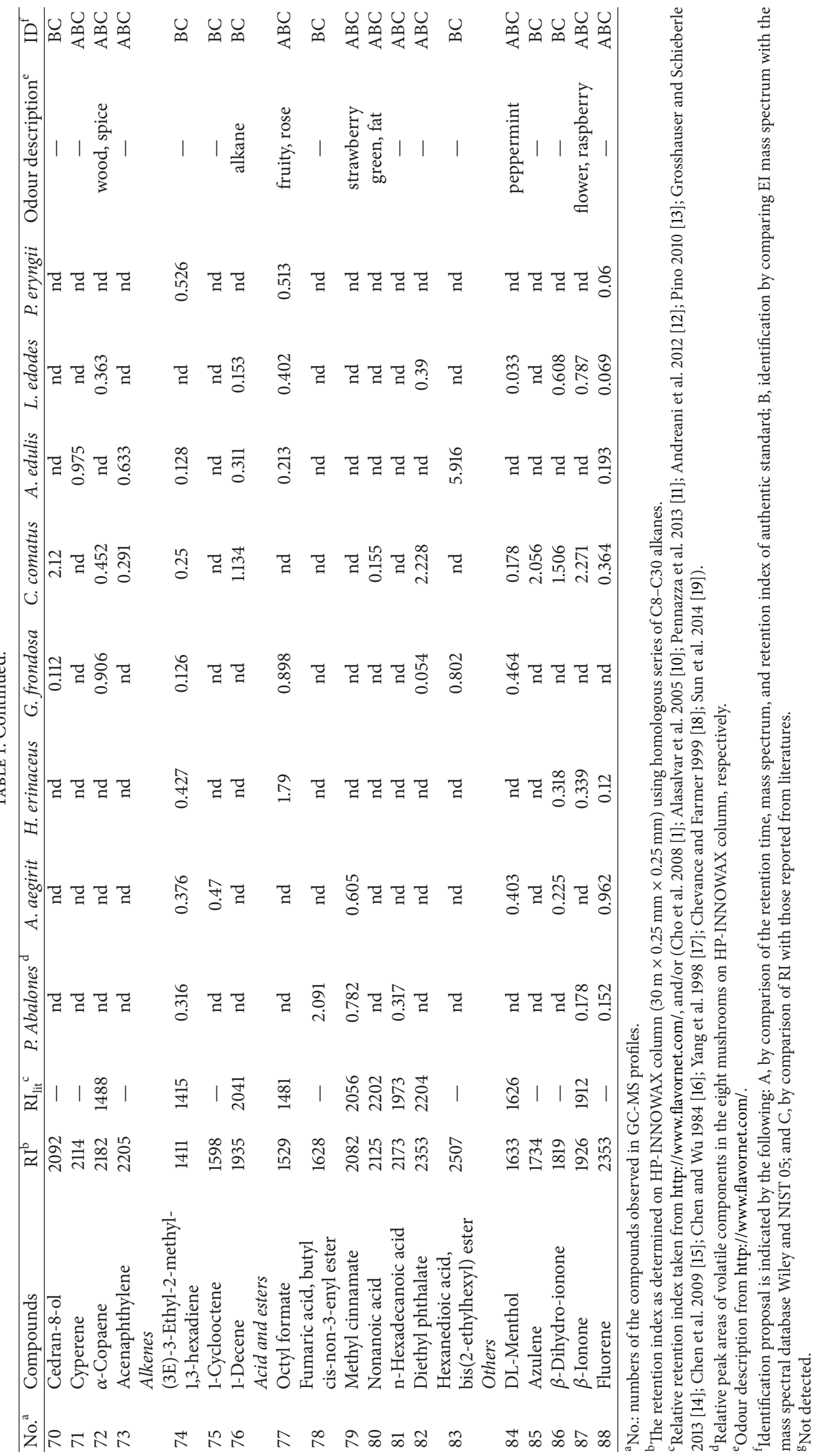




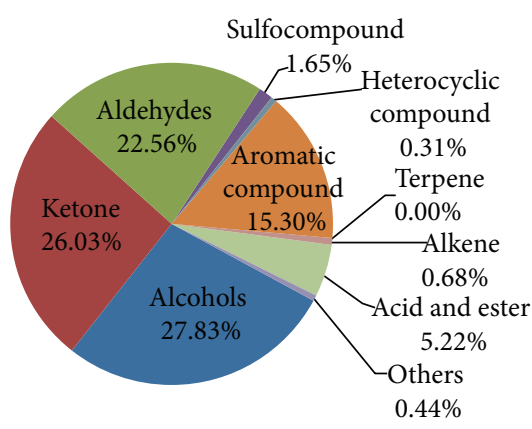

(a)

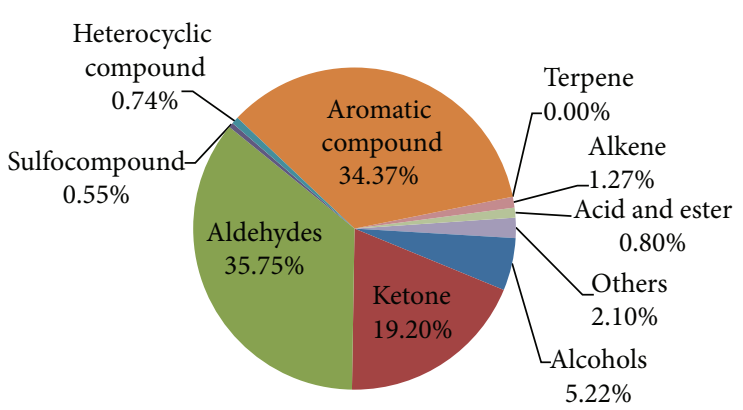

(b)

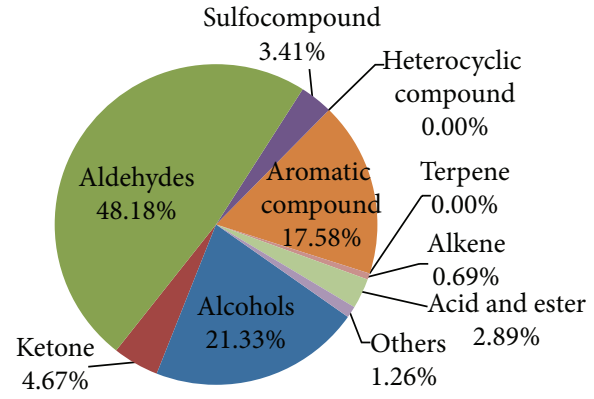

(c)

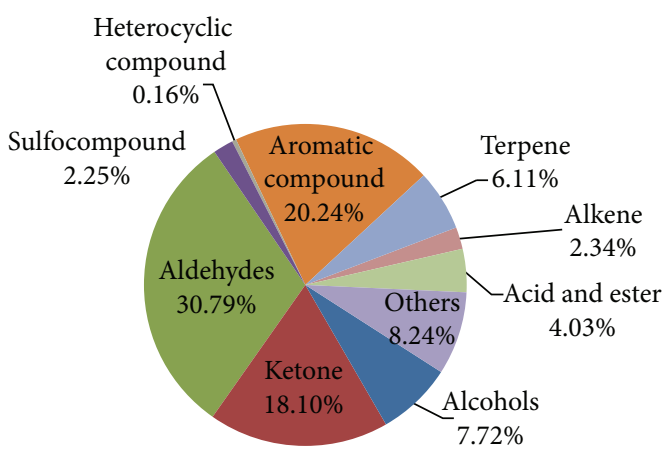

(e)

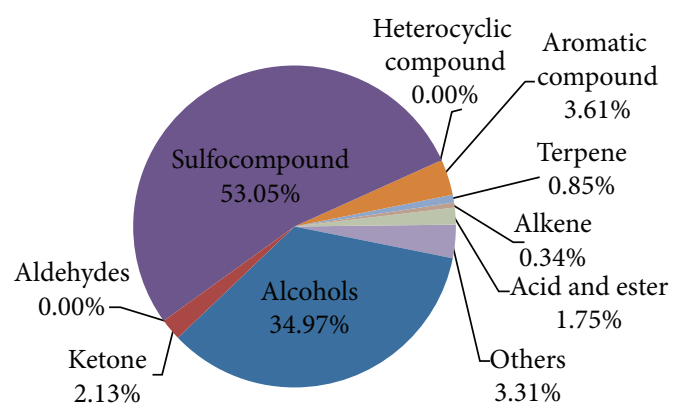

$(\mathrm{g})$

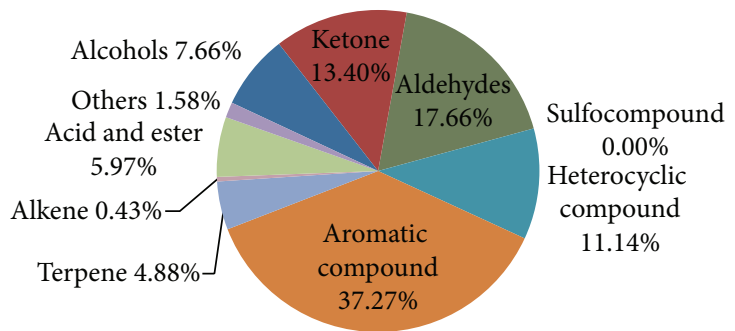

(d)

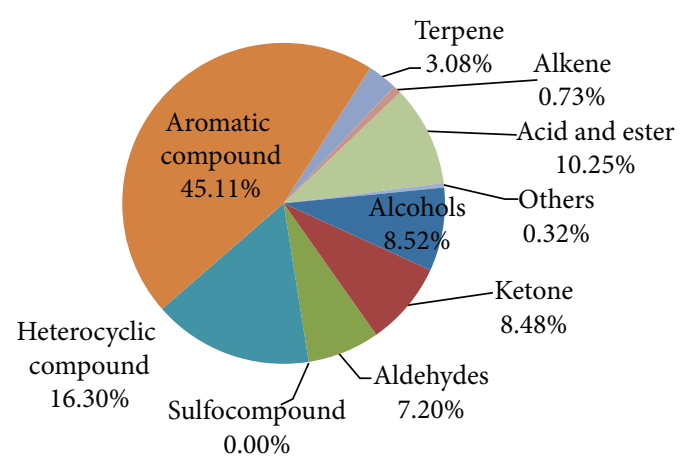

(f)

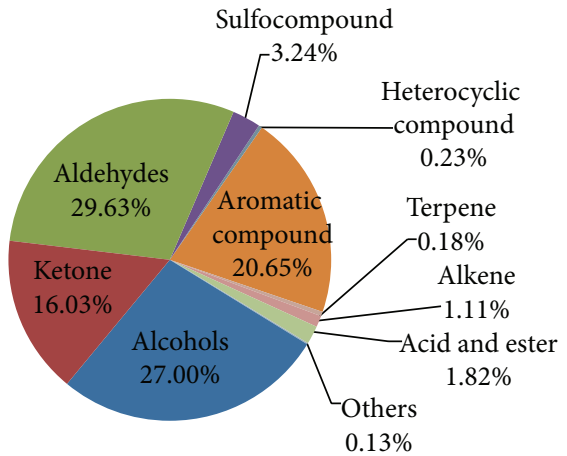

(h)

Figure 1: Percentage composition of the main groups of volatile compounds in different mushrooms. (a) Pleurotus abalonus; (b) Agrocybe aegirit; (c) Hericium erinaceus; (d) Grifola frondosa; (e) Coprinus comatus; (f) Boletus edulis; (g) Lentinula edodes; (h) Pleurotus eryngii. 
types and levels of aldehydes in different mushrooms might also be used to discriminate the mushroom species. It was noted that the dried commercial mushrooms underwent the drying process and generated some compounds such as 1octen-3-ol. The redrying process aims to offer better store samples, and steam distillation is used to extract the volatile compounds from mushroom. It is well-known that the mushroom flavor can be enhanced after cooking or heating treatment, because of the increases in the concentrations of some compounds such as 1-octen-3-ol [35]. Therefore, the "artifacts of volatile compounds" produced from Maillard reaction and lipid oxidation also are recognized as the intrinsic flavor compounds of mushroom.

Particularly, sulfur compounds were detected mainly in the flavor extract of $L$. edodes. The volatile compounds in L. edodes showed a singular intense aroma of 1, 2, 3, 5, 6pentathiepane (also named lenthionine) and a few sulfur containing degradation products of S-alkyl cysteine sulfoxide. It was also illustrated that dimethyl trisulfide, dimethyl disulfide, 1, 2, 4-trithiolane, and 1, 2, 4, 6-tetrathiepane were identified as further sulfurous metabolites in L. edodes [36]. In this study, 1, 2, 4-trithiolane was determined as the most abundant component (22.4\%) followed by 1-octen-3-ol (14.8\%) in L. edodes (Table 1).

Pyrazines, furan, and pyrroles generally provide desirable popcorn and nutty aroma to foods, obtained from Maillard reaction [37]. These three classes of chemicals were previously found in mushrooms [38] and vegetables [2] with the sensory properties of bell pepper, and peas, pungent and earthy. In the present work, 1-isoamyl-2-formyl pyrrole and 1-(2methylbutyl)-2-formyl pyrrole were determined with high concentration $(1.99 \%-4.94 \%)$ in B. edulis (Table 1).

Of the components identified, aromatic compounds were one of the important groups in all mushrooms, for example, P. abalonus (15.3\%), A. aegirit (34.4\%), H. erinaceus (17.6\%), G. frondosa (37.3\%), C. comatus (20.2), L. edodes (3.6), B. edulis (45.1\%), and P. eryngii (20.7\%). Benzaldehyde, phenyl acetaldehyde, anethole and benzeneacetic acid, and methyl ester were the most abundant components (Table 1). The existence of such a large amount of aromatic components may be the cause of "almond-like" aroma during blending of these mushrooms [16]. The high content of these compounds and their similarities in structure indicate that the aromatic compounds may have a common origin. The formation of benzaldehyde and benzyl alcohol could be increased to a significant extent if benzoic acid was blended with fresh mushrooms, suggesting that the occurrence of enzymes could be responsible for the reduction of benzoic acid or its derivative into benzaldehyde and others [16].

The small amounts of terpenes were identified in 8 mushroom species (Table 1). Eight terpene-like compounds were detected and cedran-8-ol was the most abundant terpene (2.12\%) of the total volatiles identified in C. comatus. Terpenes are often found in essential oils and contain characteristic odors [39], such as $\gamma$-muurolene, and $\alpha$-cubebene and $\beta$ cubebene were reported in the P. betulinus with the odors, which were described as wood, spice, herb, and fruit flavor [40].

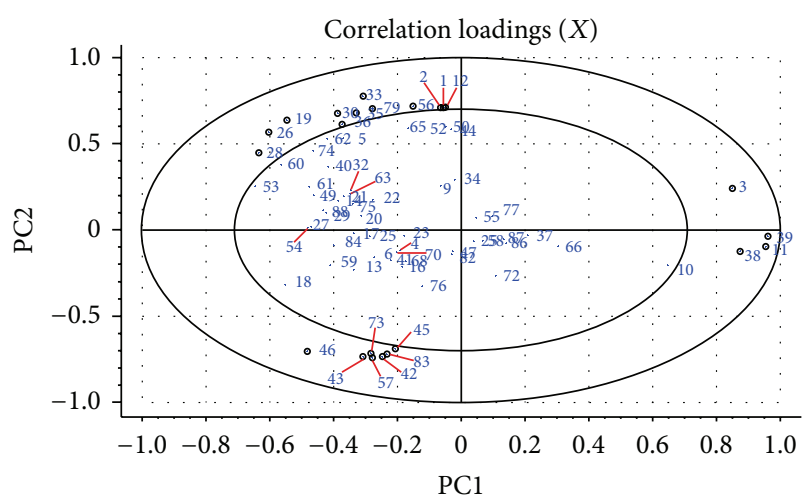

Result 1, X-expl: 40\%,21\%

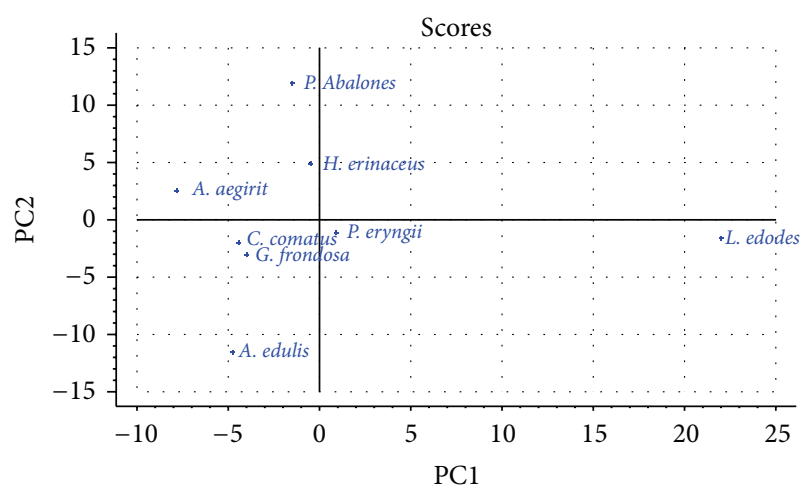

Result 1, X-expl: 40\%, 21\%

FIGURE 2: Principal components diagram of the 8 mushroom volatile compounds (variables). Ellipses represent $r^{2}=0.5$ and 1.0, respectively.

Three alkenes, for example, (3E)-3-ethyl-2-methyl-1, 3hexadiene, 1-cyclooctene, and 1-decene, were determined in the eight mushroom samples, but only 1-decene had a relative higher concentration in quantity $(1.13 \%$, Table 1$)$. Almost all alkenes could be derived from lipid degradation [41].

Table 1 also showed that 7 compounds were found in the group of acids and esters, including octyl formate, methyl cinnamate, and nonanoic acid. The acids and esters have been reported to be the major odors of fruit and grass flavors, such as octyl formate rich in blackberry with strong odors of orange fruit and rose [42]. Methyl cinnamate was reported to be abundant in the volatile compounds of pine-mushroom [1], and it was noted that this compound could prevent from the attack of the mycophagous collembolan to mushrooms [43].

An azulene-type compound was identified in C. comatus. To our best knowledge, this compound has only been isolated previously in the mushroom of Lactarius salmonicolor [44]. DL-Menthol with strong inhibitory activity against fungi of Trichoderma [45] was also detected in the tested mushrooms. This implied that some mushrooms have natural biofungicide activity.

Principal component analysis (PCA) of mushroom volatile compounds is as follows: to discriminate different mushroom species according to the GC-MS identified compounds, a PCA was performed in Figure 2, indicating 


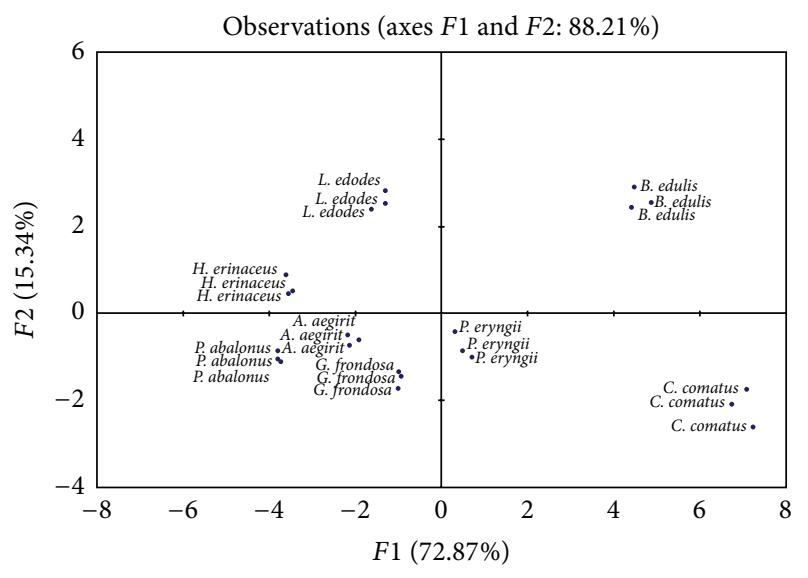

FIgURE 3: Plot of the first two principal components of the PCA model built with the electronic nose data related to the eight mushroom samples.

the projection of the GC-MS data for all samples. It was shown the differences among them. PCA provided a separation of the samples with $41 \%$ and $20 \%$ of the variation that accounted for PC1 and PC2, respectively. The contribution rate of the accumulative total of variance of the 2 factors in PCA is $61 \%$ representing that two PCs can explain $61 \%$ of the whole mushroom volatiles. The eight mushroom species were distributed according to their respective major compounds, where 3-octanol (1), 3-octanone (12), 2-octanol (2), 1-octen-3-ol (3), 1, 2, 4-trithiolane (39), octanal (28), 1octen-3-one (14), anethole (53), 6-nonenal (30), and phenyl acetaldehyde (49) had the higher power of discrimination. The first principal component (Figure 2 ) clearly separates $P$. abalonus from others due to the high content of 3-octanol (1) and 3-octanone (12). Even between Pleurotus species of $P$. abalones and P. eryngii, P. eryngii can be distinguished from others due to its high contents in hexanal (24) and 1-octen-3ol (3).

According to the abovementioned volatile profile, PCA revealed that $L$. edodes have higher content of 1-octen-3-ol (3) and 1, 2, 4-trithiolane (39), while A. aegirit have higher content of anethole (53). Meanwhile, phenyl acetaldehyde (49) and $C$. comatus have higher content of nonanal (27). Species of $H$. erinaceus was distinguished based on its 2, 4-decadienal (35) content, which was the most important differentiator compound among its volatile compounds. Another species of $B$. edulis was also separated based on higher contents in benzaldehyde (46), benzeneacetic acid, methyl ester (51), and 1-isoamyl-2-formyl pyrrole (42) (Figure 2).

3.2. Discrimination of Different Mushrooms by Electronic Nose (E-Nose). For better visualization of data, PCA was performed to identify the patterns of correlation with individual composition variables in the discrimination among different mushroom samples by using signals corresponding to three repeated exposures of each sample in Figure 3. The clearly different distributing results of different mushroom samples in PCA analysis in Figure 3 confirmed that the e-nose sensors have the ability to accurately respond to different mushrooms

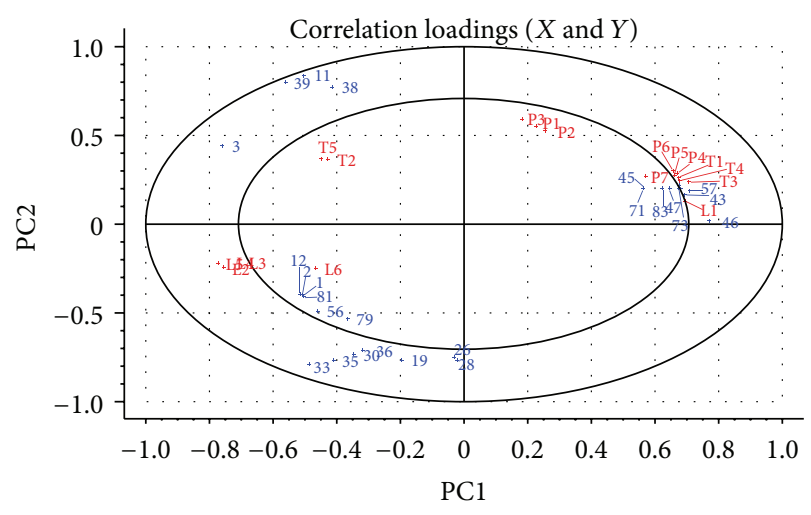

Result 1, X-expl: 34\%, 40\% Y-expl: 45\%, 7\%

(a)

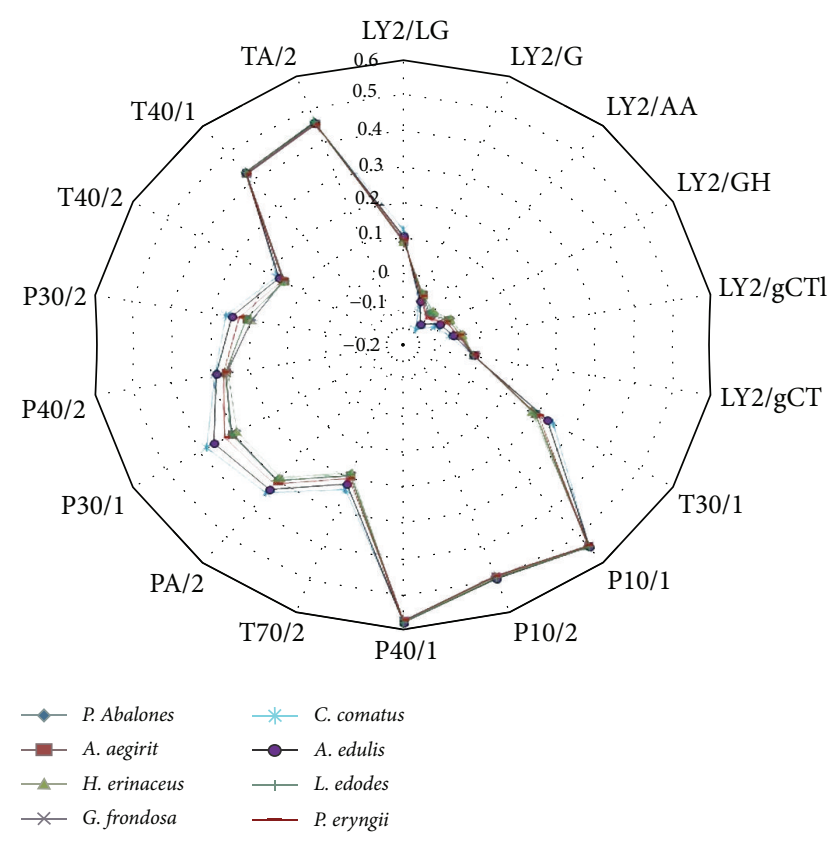

(b)

Figure 4: (a) Factor loading of PLS components 1 and 2 on GCMS data and 18 electronic nose gas sensors (L1-6 denotes LY2/LG, LY2/G, LY2/AA, LY2/GH, LY2/gCTl, and LY2/gCT, respectively; T1-5 denotes T30/1, T40/1, T40/2, T70/2, and TA/2, respectively; P1-7 denotes P10/1, P10/2, P40/1, PA/2, P30/1, P40/2, and P30/2, respectively). (b) Spider plot of the electronic nose sensors to the eight mushrooms.

with similar fingerprint chromatograms. In addition, the differences between groups have been visualized by PCA plots more clearly (Figure 3 ).

As shown in Figure 3, each group was clearly distinguished from the other groups by using PCA analysis. There was a main separation among different mushroom samples and all the mushroom samples were separated into eight groups. Even the same species such as $P$ abalonus and $P$. eryngii can be separated clearly. The score plot for the first two principal components ( $\mathrm{PC1}$ and $\mathrm{PC} 2)$ is shown in Figure 3. The score plot reveals the separation along PC1 accounting for $72.87 \%$ of the total data variance in the sample set, while 


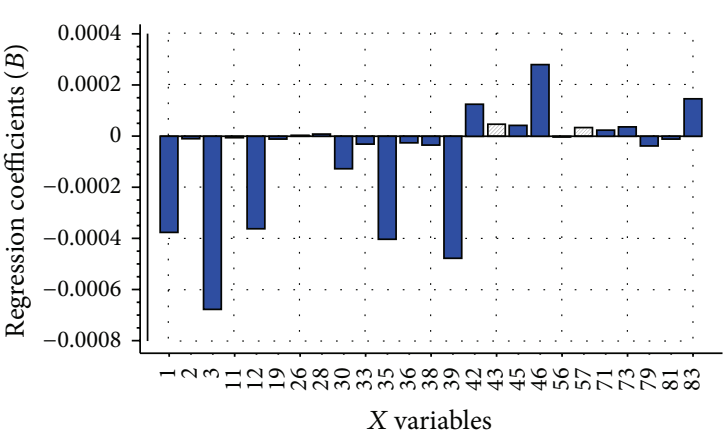

Result 1, (Y var., PC): (LY2/LG, 1) B0 $=0.101816$

(a)

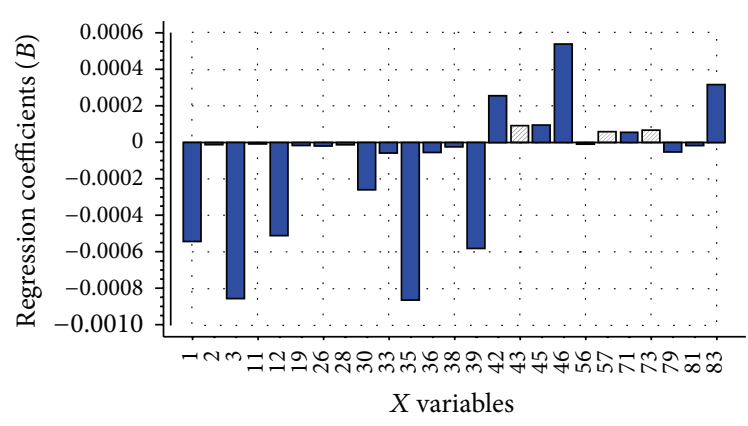

Result 1, (Y var., PC): (T70/2, 1) B0 $=0.210655$

(c)

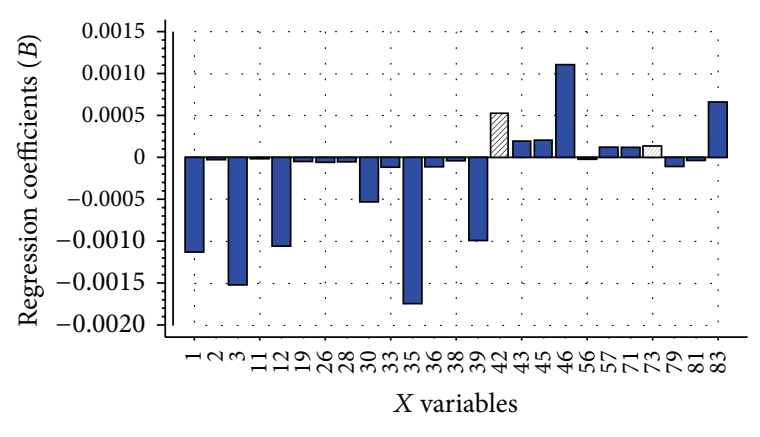

P30-1, ( $Y$ var., PC): (P30/1, 1) B0 = 0.340465

(e)

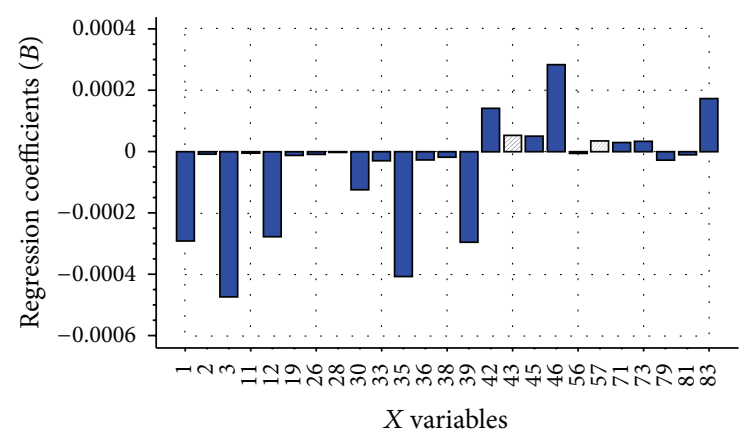

T40-2, (Y var., PC): (T40/2, 1) B0 $=0.162692$

(b)

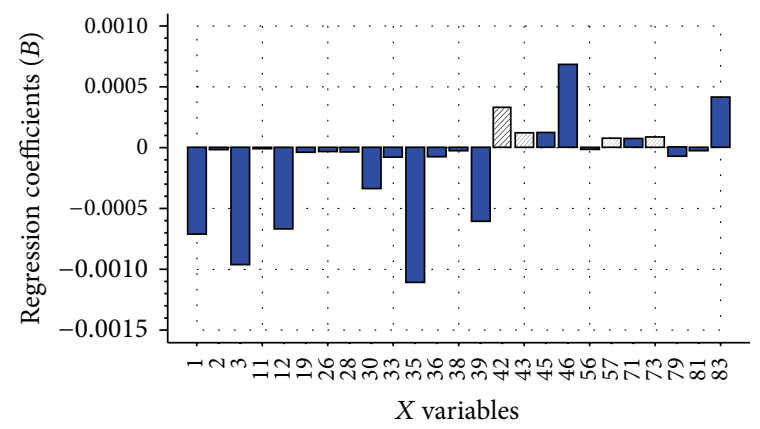

PA-2, ( $Y$ var., $P C):(P A / 2,1) B 0=0.321163$

(d)

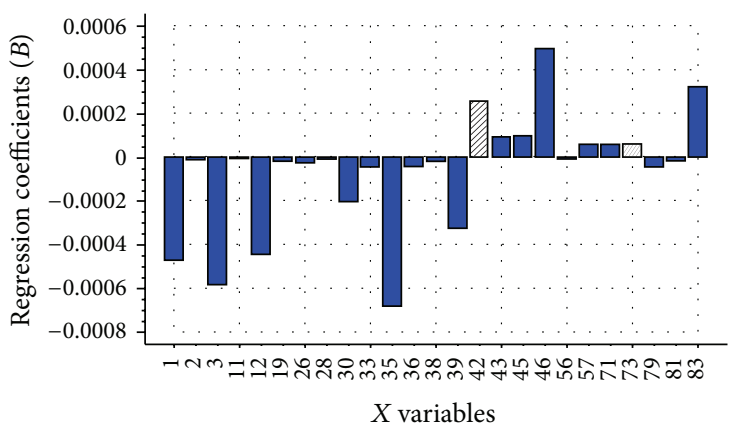

P40-2, (Y var., PC): (P40/2, 1) B0 $=0.273208$

(f)

FIGURE 5: Estimated regression coefficients and significance indications (streaked bars) from PLS1 predication models for section of gas sensors LY2/LG (a), T40/2 (b), T70/2 (c), PA/2 (d), P30/1 (e), and P40/2 (f) from GC-MS data.

separation along PC2 accounted for $15.34 \%$ of the variation in the sample set.

The results indicated that eight mushroom samples can be distinguished on the basis of different odors by e-nose with PCA method. Therefore, according to the obtained results, the e-nose can be used as a useful tool for quickly distinguishing the mushrooms, taking into account the concentration of volatile compounds. The findings from analyses carried on mushroom samples were in agreement with the results obtained by means of GC-MS as they both separated mushroom samples successfully. Hence, e-nose could be used as an identification tool for mushroom.
3.3. Relationship between GC-MS Profiles and E-Nose Analysis. To study relationships between GC-MS data and e-nose responses, two data sets were analyzed by PLS. The GC-MS data were selected from all GC-MS profiles, according to the results in Figure 2, which located in the ring. Figure 4(a) shows two factors loading plot for GC-MS data ( $X$-matrix) and 18 sensors ( $Y$-matrix). Four sensors clusters located in three quadrants and 25 GC-MS peaks are placed in three locations. The derived PLSR model included two significant PCs successfully explaining $74 \%$ of the cross validated variance. Figure 4(b) indicates a typical response of the e-nose sensors for the measurement of the eight mushroom samples. 
Each curve represented the maximum response value of the mushroom volatiles on the sensors during parsing time. Although they had a roughly identical trend on the 18 sensors, there were significant differences between eight mushrooms on sensors, for instance, P30/2, P40/2, P30/1, and PA/2.

To further investigate the compounds with the greater contribution to e-nose sensors, PLS1 regression analysis was carried out. Figure 5 shows the results from the PLS1 regression analysis for the contribution of GC-MS profiles to the sensors response data LY2/LG, T40/2, T70/2, PA/2, $\mathrm{P} 30 / 1$, and P40/2. Streaky bars demonstrated significant GC-MS compounds. In general, enormous volatiles were negatively related to selected sensors by means of their regression coefficients in PLS regression modeling. Merely two compounds, 1-(2-methylbutyl)-2-formyl pyrrole (43) and $\mathrm{N}$-ethylpropionanilide (57), appeared to possess significant sensitivity and explained $78 \%$ and $70 \%$ of the variation in LY2/LG (A) and T40/2 (B), respectively. Three compounds, 1-(2-methylbutyl)-2-formyl pyrrole (43), Nethylpropionanilide (57), and acenaphthylene (73), illustrated significant sensitivity and explained $68 \%$ of variation in T70/2 (C). Four compounds, 1-isoamyl-2-formyl pyrrole (42), 1-(2methylbutyl)-2-formyl pyrrole (43), N-ethylpropionanilide (57), and acenaphthylene (73), suggested significant sensitivity and explained $64 \%$ of variation in PA/2 (D). Two compounds, 1-isoamyl-2-formyl pyrrole (42) and acenaphthylene (73), had significant sensitivity and explained $64 \%$ and $62 \%$ of the variation in P30/1 (E) and P40/2 (F), respectively. The above results were similar to the conclusions in literature [4650]. The object substances for LY/LG were oxynitride and sulfide, and P30/1 was hydrocarbons and ammonia, and PA/2 was ammonia and amine compounds, and so forth.

\section{Conclusions}

In this study, it was the first time that the volatile compounds of 8 different edible mushroom species were characterized by using both GC-MS and e-nose. Based on the GC-MS analysis, a total of 88 volatile compounds were identified and differences in the composition of volatile components from eight mushrooms were observed. It was feasible to classify the mushroom samples into eight groups by using GC-MS and enose. Elementary results confirmed the usefulness of GC-MS and electronic nose for classification purpose of mushroom. This combination for the volatile analysis with chemometric methods can be applied to distinguish different mushrooms successfully. Furthermore, this study results about the volatile composition of commercial mushrooms could help to set up a finger spectrum by e-nose to identify the species of edible fungi.

\section{Conflict of Interests}

The authors declare that there is no conflict of interests regarding the publication of this paper.

\section{Acknowledgments}

The authors thank Mr. Jiancai Zhu for his technical assistance in instrumental analysis and data acquisition. This work was financially supported by the seed fund of Shanghai Institute of Technology.

\section{References}

[1] I. H. Cho, H. J. Namgung, H. K. Choi, and Y. S. Kim, "Volatiles and key odorants in the pileus and stipe of pine-mushroom (Tricholoma matsutake Sing.)," Food Chemistry, vol. 106, no. 1, pp. 71-76, 2008.

[2] S. Jong and J. Birmingham, "Mushrooms as a source of natural flavor and aroma compounds," in Mushroom Biology and Mushroom Products, S. T. Chang, J. A. Buswell, and S. W. Chiu, Eds., pp. 345-366, The Chinese University Press, Hong Kong, 1993.

[3] A. Gallois, B. Gross, D. Langlois, H.-E. Spinnler, and P. Brunerie, "Influence of culture conditions on production of flavour compounds by 29 ligninolytic basidiomycetes," Mycological Research, vol. 94, no. 4, pp. 494-504, 1990.

[4] S. L. Cuppett, A. M. Parkhurst, W. Chung, M. Weyer, and L. B. Bullerman, "Factors affecting sensory attributes of oyster mushrooms," Journal of Food Quality, vol. 21, no. 5, pp. 383-395, 1998.

[5] S. Murahashi, "Uber die riechstoffe des matutake," Scientific Papers of the Institute of Physical and Chemical Research, vol. 34, pp. 155-172, 1938.

[6] W. Grosch, "Determination of potent odourants in foods by aroma extract dilution analysis (AEDA) and calculation of odour activity values (OAVs)," Flavour and Fragrance Journal, vol. 9, no. 4, pp. 147-158, 1994.

[7] F. Maggi, T. Bílek, D. Lucarini, F. Papa, G. Sagratini, and S. Vittori, "Melittis melissophyllum L. subsp. melissophyllum (Lamiaceae) from central Italy: a new source of a mushroom-like flavour," Food Chemistry, vol. 113, no. 1, pp. 216-221, 2009.

[8] I. H. Cho, S. M. Lee, S. Y. Kim, H.-K. Choi, K.-O. Kim, and Y.-S. Kim, "Differentiation of aroma characteristics of pinemushrooms (Tricholoma matsutake Sing.) of different grades using gas chromatography-olfactometry and sensory analysis," Journal of Agricultural and Food Chemistry, vol. 55, no. 6, pp. 2323-2328, 2007.

[9] R. Malheiro, P. G. de Pinho, S. Soares, A. C. da Silva Ferreira, and P. Baptista, "Volatile biomarkers for wild mushrooms species discrimination," Food Research International, vol. 54, no. 1, pp. 186-194, 2013.

[10] C. Alasalvar, K. D. A. Taylor, and F. Shahidi, "Comparison of volatiles of cultured and wild sea bream (Sparus aurata) during storage in ice by dynamic headspace analysis/gas chromatography-mass spectrometry," Journal of Agricultural and Food Chemistry, vol. 53, no. 7, pp. 2616-2622, 2005.

[11] G. Pennazza, C. Fanali, M. Santonico et al., "Electronic nose and GC-MS analysis of volatile compounds in Tuber magnatum Pico: evaluation of different storage conditions," Food Chemistry, vol. 136, no. 2, pp. 668-674, 2013.

[12] S. Andreani, T. Barboni, J.-M. Desjobert, J. Paolini, J. Costa, and A. Muselli, "Essential oil composition and chemical variability of Xanthium italicum Moretti from Corsica," Flavour and Fragrance Journal, vol. 27, no. 3, pp. 227-236, 2012.

[13] J. A. Pino, "Volatile compounds of Cassia grandis L. f. Fruit from Cuba," Journal of Essential Oil Research, vol. 22, no. 6, pp. 599$601,2010$. 
[14] S. Grosshauser and P. Schieberle, "Characterization of the key odorants in pan-fried white mushrooms (Agaricus bisporus L.) by means of molecular sensory science: comparison with the raw mushroom tissue," Journal of Agricultural and Food Chemistry, vol. 61, no. 16, pp. 3804-3813, 2013.

[15] G. Chen, H. Song, and C. Ma, "Aroma-active compounds of Beijing roast duck," Flavour and Fragrance Journal, vol. 24, no. 4, pp. 186-191, 2009.

[16] C.-C. Chen and C.-M. Wu, "Volatile components of mushroom (Agaricus subrufecens)," Journal of Food Science, vol. 49, no. 4, pp. 1208-1209, 1984.

[17] M.-S. Yang, C.-C. Chyau, D.-T. Horng, and J.-S. Yang, "Effects of irradiation and drying on volatile components of fresh shiitake (Lentinus edodesSing)," Journal of the Science of Food and Agriculture, vol. 76, no. 1, pp. 72-76, 1998.

[18] F. F. V. Chevance and L. J. Farmer, "Identification of major volatile odor compounds in frankfurters," Journal of Agricultural and Food Chemistry, vol. 47, no. 12, pp. 5151-5160, 1999.

[19] L. Sun, J. Chen, M. Li, Y. Liu, and G. Zhao, "Effect of star anise (Illicium verum) on the volatile compounds of stewed chicken," Journal of Food Process Engineering, vol. 37, no. 2, pp. 131-145, 2014.

[20] B. Wang, S. Xu, and D.-W. Sun, "Application of the electronic nose to the identification of different milk flavorings," Food Research International, vol. 43, no. 1, pp. 255-262, 2010.

[21] T. Feng, H. Zhuang, R. Ye, Z. Jin, X. Xu, and Z. Xie, "Analysis of volatile compounds of Mesona Blumes gum/rice extrudates via GC-MS and electronic nose," Sensors and Actuators B: Chemical, vol. 160, no. 1, pp. 964-973, 2011.

[22] H. Cheng, Z. H. Qin, X. F. Guo, X. S. Hu, and J. H. Wu, "Geographical origin identification of propolis using GC-MS and electronic nose combined with principal component analysis," Food Research International, vol. 51, no. 2, pp. 813-822, 2013.

[23] F. Maggi, F. Papa, G. Cristalli, G. Sagratini, and S. Vittori, "Characterisation of the mushroom-like flavour of Melittis melissophyllum L. subsp. melissophyllum by headspace solid-phase microextraction (HS-SPME) coupled with gas chromatography (GC-FID) and gas chromatography-mass spectrometry (GCMS)," Food Chemistry, vol. 123, no. 4, pp. 983-992, 2010.

[24] C. Cevoli, L. Cerretani, A. Gori, M. F. Caboni, T. Gallina Toschi, and A. Fabbri, "Classification of Pecorino cheeses using electronic nose combined with artificial neural network and comparison with GC-MS analysis of volatile compounds," Food Chemistry, vol. 129, no. 3, pp. 1315-1319, 2011.

[25] S. Song, X. Zhang, K. Hayat et al., "Correlating chemical parameters of controlled oxidation tallow to gas chromatographymass spectrometry profiles and e-nose responses using partial least squares regression analysis," Sensors and Actuators B: Chemical, vol. 147, no. 2, pp. 660-668, 2010.

[26] X. Wang, M. Zhao, R. Ju et al., "Visualizing quantitatively the freshness of intact fresh pork using acousto-optical tunable filter-based visible/near-infrared spectral imagery," Computers and Electronics in Agriculture, vol. 99, pp. 41-53, 2013.

[27] E. Combet, J. Henderson, D. C. Eastwood, and K. S. Burton, "Eight-carbon volatiles in mushrooms and fungi: properties, analysis, and biosynthesis," Mycoscience, vol. 47, no. 6, pp. 317326, 2006.

[28] S. Assaf, Y. Hadar, and C. G. Dosoretz, "1-Octen-3-ol and 13hydroperoxylinoleate are products of distinct pathways in the oxidative breakdown of linoleic acid by Pleurotus pulmonarius," Enzyme and Microbial Technology, vol. 21, no. 7, pp. 484-490, 1997.
[29] C. Prost, T. Serot, and M. Demaimay, "Identification of the most potent odorants in wild and farmed cooked turbot (Scophtalamus maximus L.)," Journal of Agricultural and Food Chemistry, vol. 46, no. 8, pp. 3214-3219, 1998.

[30] S. Baldermann, M. Naim, and P. Fleischmann, "Enzymatic carotenoid degradation and aroma formation in nectarines (Prunus persica)," Food Research International, vol. 38, no. 8-9, pp. 833-836, 2005.

[31] A. C. Silva Ferreira, J. Monteiro, C. Oliveira, and P. Guedes de Pinho, "Study of major aromatic compounds in port wines from carotenoid degradation," Food Chemistry, vol. 110, no. 1, pp. 8387, 2008.

[32] C.-T. Ho and Q. Chen, "Lipids in food flavors: an overview," in Lipids in Food Flavors, vol. 558 of ACS Symposium Series, pp. 214, American Chemical Society, Washington, DC, USA, 1994.

[33] M. van Praag, H. S. Stein, and M. S. Tibbetts, "Steam volatile aroma constituents of roasted cocoa beans," Journal of Agricultural and Food Chemistry, vol. 16, no. 6, pp. 1005-1008, 1968.

[34] D. B. Josephson and R. C. Lindsay, "Enzymic generation of volatile aroma compounds from fresh fish," in Biogeneration of Aromas, vol. 317 of ACS Symposium Series, pp. 201-219, American Chemical Society, Washington, DC, USA, 1986.

[35] J. A. Maga, "Influence of maturity, storage and heating on the flavor of mushroom (Agaricusbisporus) caps and stems," Journal of Food Processing and Preservation, vol. 5, no. 2, pp. 95-101, 1981.

[36] M. Fraatz and H. Zorn, "Fungal flavours," in Industrial Applications: The Mycota, M. Hofrichter, Ed., vol. 10, pp. 249-268, Springer, Berlin, Germany, 2011.

[37] K. Eric, L. V. Raymond, S. Abbas et al., "Temperature and cysteine addition effect on formation of sunflower hydrolysate Maillard reaction products and corresponding influence on sensory characteristics assessed by partial least square regression," Food Research International, vol. 57, pp. 242-258, 2014.

[38] J. A. Maga, "Mushroom flavor," Journal of Agricultural and Food Chemistry, vol. 29, no. 1, pp. 1-4, 1981.

[39] K. Bauer, D. Garbe, and H. Surburg, Common Fragrance and Flavor Materials, John Wiley \& Sons, 2008.

[40] J. Rösecke, M. Pietsch, and W. A. König, "Volatile constituents of wood-rotting basidiomycetes," Phytochemistry, vol. 54, no. 8, pp. 747-750, 2000.

[41] E. Selke, W. K. Rohwedder, and H. J. Dutton, "Volatile components from triolein heated in air," Journal of the American Oil Chemists' Society, vol. 54, no. 2, pp. 62-67, 1977.

[42] K. Klesk and M. Qian, "Preliminary aroma comparison of Marion (Rubus spp. hyb) and evergreen ( $R$. laciniatus L.) blackberries by dynamic headspace/OSME technique," Journal of Food Science, vol. 68, no. 2, pp. 697-700, 2003.

[43] T. Sawahata, S. Shimano, and M. Suzuki, "Tricholoma matsutake 1-Ocen-3-ol and methyl cinnamate repel mycophagous Proisotoma minuta (Collembola: Insecta)," Mycorrhiza, vol. 18, no. 2, pp. 111-114, 2008.

[44] G. Athanasakis, N. Aligiannis, Z. Gonou-Zagou, A.-L. Skaltsounis, and N. Fokialakis, "Antioxidant properties of the wild edible mushroom lactarius salmonicolor," Journal of Medicinal Food, vol. 16, no. 8, pp. 760-764, 2013.

[45] M. Soković and L. J. L. D. van Griensven, "Antimicrobial activity of essential oils and their components against the three major pathogens of the cultivated button mushroom, Agaricus bisporus," European Journal of Plant Pathology, vol. 116, no. 3, pp. 211-224, 2006. 
[46] Z. Qin, X. Pang, D. Chen, H. Cheng, X. Hu, and J. Wu, "Evaluation of Chinese tea by the electronic nose and gas chromatography-mass spectrometry: correlation with sensory properties and classification according to grade level," Food Research International, vol. 53, no. 2, pp. 864-874, 2013.

[47] X. Wu, L. Ye, K. Liu et al., "Antibacterial properties of mesoporous copper-doped silica xerogels," Biomedical Materials, vol. 4, no. 4, Article ID 045008, 2009.

[48] X. Wu, J. Wei, X. Lu et al., "Chemical characteristics and hemostatic performances of ordered mesoporous calcium-doped silica xerogels," Biomedical Materials, vol. 5, no. 3, Article ID 035006, 2010.

[49] H. Zhou, X. Wu, J. Wei et al., "Stimulated osteoblastic proliferation by mesoporous silica xerogel with high specific surface area," Journal of Materials Science: Materials in Medicine, vol. 22, no. 3, pp. 731-739, 2011.

[50] X. Wu and S. Wang, "Regulating MC3T3-E1 cells on deformable poly( $\varepsilon$-caprolactone) honeycomb films prepared using a surfactant-free breath figure method in a water-miscible solvent," ACS Applied Materials and Interfaces, vol. 4, no. 9, pp. 4966-4975, 2012. 

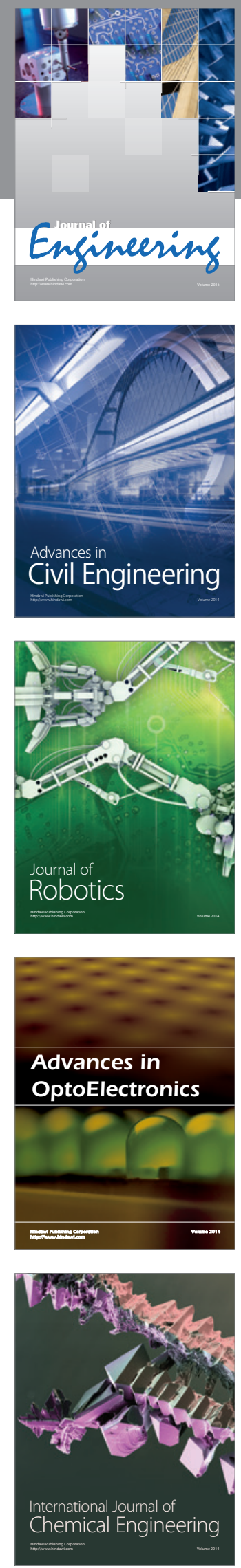

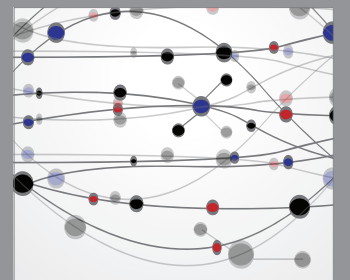

The Scientific World Journal
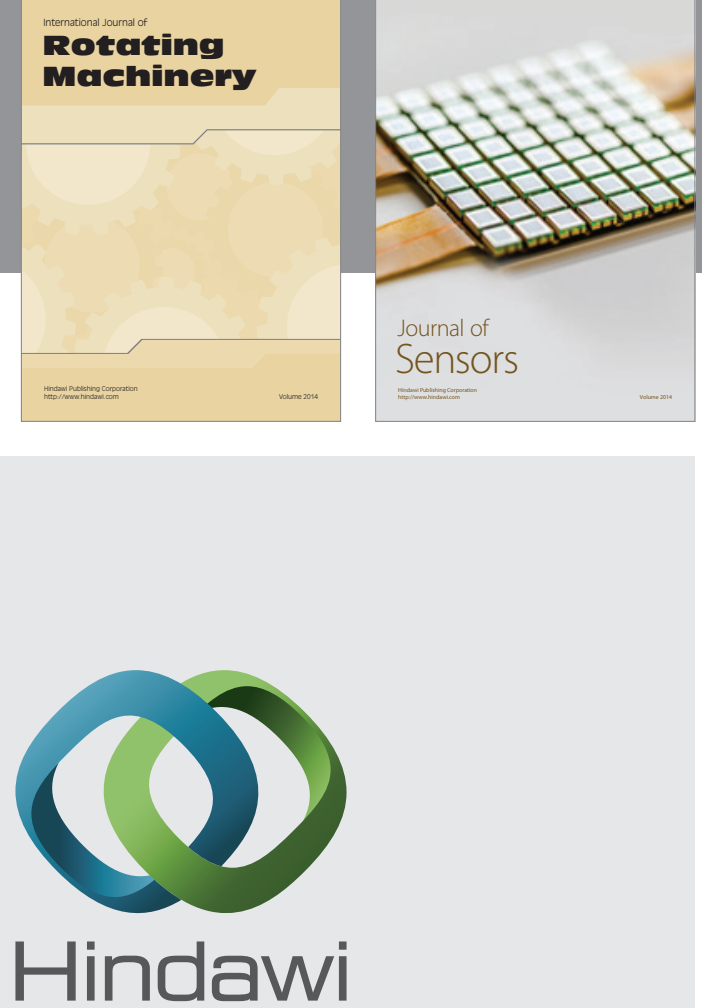

Submit your manuscripts at http://www.hindawi.com
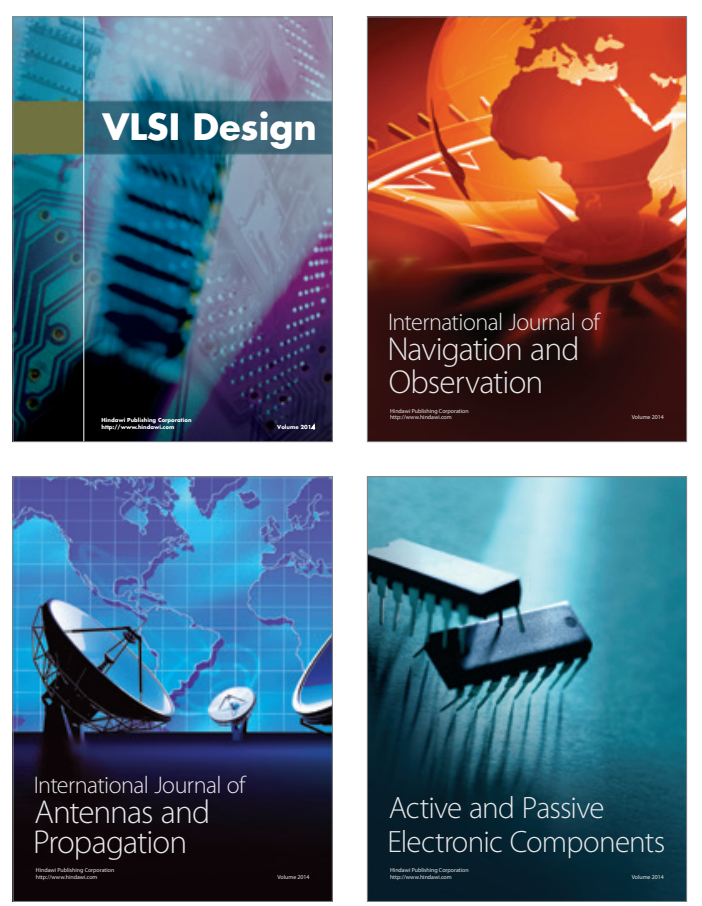
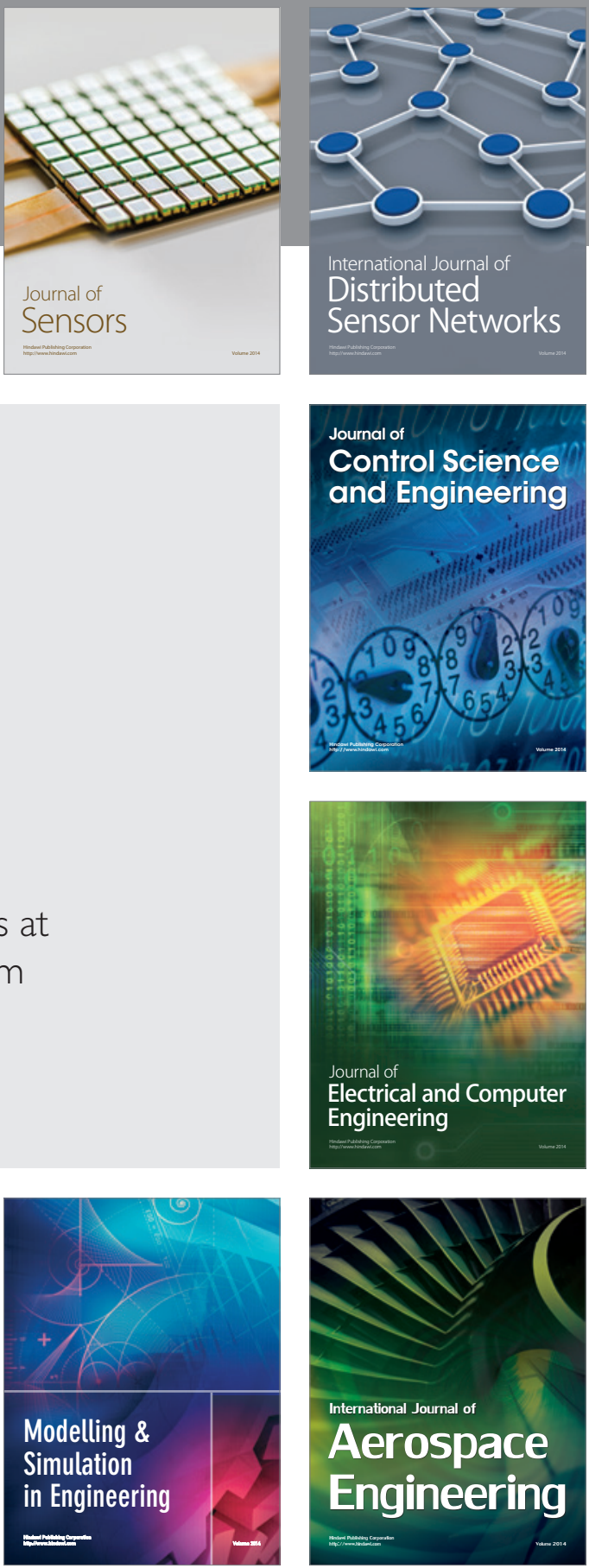

Journal of

Control Science

and Engineering
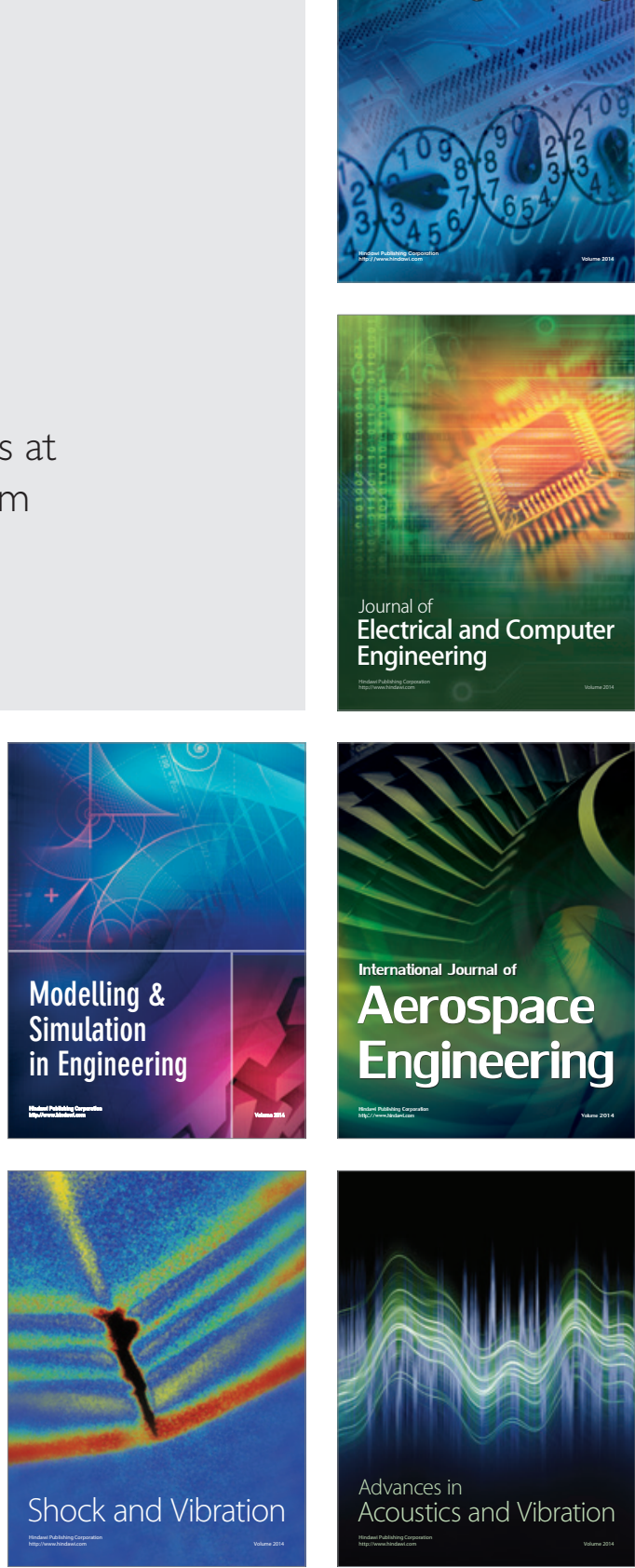